\title{
Markov multi-variate survival indicators for default simulation as a new characterization of the Marshall-Olkin law
}

\author{
Damiano Brigo \\ Dept of Mathematics, Imperial College London, \\ 180 Queen's Gate, London, UK, \\ email: damiano.brigo@imperial.ac.uk \\ Jan-Frederik Mai \\ XAIA Investment $\mathrm{GmbH}$, \\ Sonnenstr. 19, 80331 München, Germany, \\ email: jan-frederik.mai@xaia.com, phone: +49(0)89-589275-131

\section{Matthias Scherer} \\ Chair of Mathematical Finance, \\ Technische Universität München, \\ Parkring 11, 85748 Garching-Hochbrück, Germany, \\ email: scherer@tum.de, phone: +49(0)89-289-17402
}

\begin{abstract}
A new characterization of the Marshall-Olkin distribution is provided: all subvectors of the associated survival indicators are continuous-time Markov chains. This property is crucial to overcome practical limitations for the modeling of highdimensional default times (rebalancing, iterative simulation, consistent sub-portfolios).
\end{abstract}

Classification Codes: AMS 60E07, 62H05, 62H20, 62H99; JEL C15, C16.

Keywords: Stepwise default simulation, default-risk modeling, default dependence, portfolio credit risk, Marshall-Olkin distribution, nested margining property. 


\section{Introduction}

\section{Introduction}

Implementing the simulation of a global portfolio -for the computation of certain risk measures, counterparty exposures, or pricing of valuation adjustments- is a numerically intensive task faced by all financial institutions, see [Brigo et al. (2013)]. An integral component of such an economic scenario generator is a model for the vector of default times $\left(\tau_{1}, \ldots, \tau_{d}\right)$ of all credit-risky assets, with dimension $d$ easily in the thousands. In practice, the model cosmos for the vector of default times is limited to "simple" models, since a high level of practical viability needs to be guaranteed. This explains why copula-based models are often the industry's preferred choice, cf. [McNeil et al. (2005)]. In many real-world applications one runs a simulation along a pre-specified time grid, and simulation of the dependence structure needs to be aligned with the relevant time steps. Moreover, being able to deal with sub-portfolios consistently with the full model and its simulation, in that removing a component at a default or portfolio rebalancing event does not change the probability law, is also convenient in practice. We term this fundamental property "nested margining" and show that it appears natural in the context of the Marshall-Olkin law, which the present article advocates as a model for $\left(\tau_{1}, \ldots, \tau_{d}\right)$. We argue that this choice is by no means arbitrary, but rather stems from a number of practical limitations indicated above and discussed in depth in Section 3. Mathematically, this corresponds to a need for a Markovianity property that constitutes a novel characterization of the Marshall-Olkin law. To this end, the remaining sections of the paper are organized as follows:

Mathematical contribution: Section 2 shows that the Marshall-Olkin distribution is characterized by the property that all subvectors of its associated survival indicator process are continuous-time Markov chains. This result demonstrates that a need for Markovianity on all sub-portfolio levels naturally limits the model cosmos to the (seemingly small) class of Marshall-Olkin distributions. This statement formally completes an initial investigation in [Brigo, Chourdakis (2012)], where Markovianity of minima of default times is investigated and linked to extreme-value copulas under a weaker notion of lack-of-memory.

Real world restrictions: Section 3 provides a collection of practical restrictions regarding the modeling of multiple defaults for company-wide tasks as mentioned above. Besides the necessity for stepwise simulation along a given time grid, a "nested margining property" is required in order to cope with occurred defaults and portfolio rebalancing tasks. As a consequence of all restrictions, it is argued that a reasonable trade-off between applicability and model flexibility might consist in modeling the multi-variate survival indicator process such that each subvector of it is a continuous-time Markov chain. In conjunction with Section 2 this renders the Marshall-Olkin distribution the natural and indeed unique choice for modeling default times.

Implementation advice: Having identified the Marshall-Olkin distribution as essentially the only model satisfying our list of practical demands, we find it appropriate to scrutinize some recent statistical literature on the efficient parameterization and stepwise 
simulation of the Marshall-Olkin distribution, with an eye towards a feasible implementation via the so-called Lévy-frailty construction. This is done in Section 4.

\section{A new characterization of the Marshall-Olkin law}

We denote the survival indicator process of the considered default times $\left(\tau_{1}, \ldots, \tau_{d}\right)$ by $\mathbf{Z}(t):=\left(\mathbf{1}_{\left\{\tau_{1}>t\right\}}, \ldots, \mathbf{1}_{\left\{\tau_{d}>t\right\}}\right)$. Furthermore, we denote by $\mathbf{Z}_{I}$ the $|I|$-margin of $\mathbf{Z}$, which only consists of the components indexed by $I \subset\{1, \ldots, d\}$, so that, in particular, $\boldsymbol{Z}=\boldsymbol{Z}_{\{1, \ldots, d\}}$. It will be convenient to identify the state space $\{0,1\}^{d}$ of $\mathbf{Z}$ with the power set of $\{1, \ldots, d\}$ via the bijection

$$
h(I):=\left(\mathbf{1}_{\{1 \in I\}}, \ldots, \mathbf{1}_{\{d \in I\}}\right), \quad I \subset\{1, \ldots, d\} .
$$

The following distribution is the essential object of interest in what follows.

\section{Definition 2.1 (The Marshall-Olkin distribution)}

On a probability space $(\Omega, \mathcal{F}, \mathbb{P})$, a random vector $\left(\tau_{1}, \ldots, \tau_{d}\right)$ taking values in $[0, \infty)^{d}$ is said to follow a Marshall-Olkin distribution (MO) if it has the survival function

$$
\mathbb{P}\left(\tau_{1}>t_{1}, \ldots, \tau_{d}>t_{d}\right)=\exp \left(-\sum_{\emptyset \neq I \subset\{1, \ldots, d\}} \lambda_{I} \max _{i \in I}\left\{t_{i}\right\}\right), \quad t_{1}, \ldots, t_{d} \geq 0,
$$

for non-negative parameters $\left\{\lambda_{I}\right\}, \emptyset \neq I \subset\{1, \ldots, d\}$, satisfying $\sum_{I: i \in I} \lambda_{I}>0$ for all $i=$ $1, \ldots, d$. A canonical construction for this distribution, due to [Marshall, Olkin (1967)], is based on a collection of independent exponential random variables $\left\{E_{I}\right\}_{\emptyset \neq I \subset\{1, \ldots, d\}}$ with rates $\lambda_{I}$ and given via

$$
\tau_{i}:=\min \left\{E_{I}: i \in I\right\}, \quad i=1, \ldots, d,
$$

where $\lambda_{I}=0$ is conveniently interpreted as $E_{I} \equiv \infty$.

The following theorem characterizes the Marshall-Olkin law of the vector $\left(\tau_{1}, \ldots, \tau_{d}\right)$ in terms of Markov properties of the processes $\mathbf{Z}_{I}$. The sufficiency statement is intuitive, since the Markov property of the $\mathbf{Z}_{I}$ 's can be shown to imply the so-called lack-ofmemory property for $\left(\tau_{1}, \ldots, \tau_{d}\right)$, which is known to characterize the Marshall-Olkin law at least since the pioneering work of [Marshall, Olkin (1967)]. The necessity statement is maybe less intuitive but implies important limitations for practical modeling, cf. Section 3. Its proof is based on an alternative stochastic construction of the MarshallOlkin law from [Arnold (1975)], which shows that a dynamic simulation of $\mathbf{Z}_{I}$ is actually Markovian.

\section{Theorem 2.2 (Markovianity of survival indicators)}

$\left(\tau_{1}, \ldots, \tau_{d}\right)$ has a Marshall-Olkin distribution $\Leftrightarrow$ the $|I|$-dimensional survival indicator processes $\mathbf{Z}_{I}$ are continuous time Markov chains for all subsets $\emptyset \neq I \subset\{1, \ldots, d\}$. 


\section{Proof}

" $\Leftarrow$ " By the time-homogeneous Markov property of $\mathbf{Z}=\mathbf{Z}_{\{1, \ldots, d\}}$, there is a transition function $p_{\mathbf{x}, \mathbf{y}}(t)$ for $\mathbf{x}, \mathbf{y} \in\{0,1\}^{d}$ and $t \geq 0$ such that

$$
\mathbb{P}\left(\mathbf{Z}\left(t_{n}\right)=\mathbf{x}_{n}, \ldots, \mathbf{Z}\left(t_{1}\right)=\mathbf{x}_{1}\right)=p_{(1, \ldots, 1), \mathbf{x}_{1}}\left(t_{1}\right) \prod_{\ell=2}^{n} p_{\mathbf{x}_{\ell-1}, \mathbf{x}_{\ell}}\left(t_{\ell}-t_{\ell-1}\right)
$$

for $t_{n}>\ldots>t_{1}>0$ and $\mathbf{x}_{1}, \ldots, \mathbf{x}_{n} \in\{0,1\}^{d}$. Let $t, s_{1}, \ldots, s_{d} \geq 0$ be arbitrary and denote by $\pi$ a permutation such that $s_{\pi(1)} \leq s_{\pi(2)} \leq \ldots \leq s_{\pi(d)}$ is the ordered list of $s_{1}, \ldots, s_{d}$. Define the following subsets of $\{0,1\}^{d}$ :

$$
A_{1}:=\{(1, \ldots, 1)\}, \quad A_{k}:=\left\{\mathbf{x} \in\{0,1\}^{d}: x_{\pi(\ell)}=1 \text { for all } \ell \geq k\right\}, \quad k=2, \ldots, d .
$$

In words, $A_{k}$ denotes the subset of $\{0,1\}^{d}$ in which all components $\pi(k), \ldots, \pi(d)$ are still alive. There is a finite number $N$ of distinct paths $\left(\mathbf{x}_{2}^{(i)}, \ldots, \mathbf{x}_{d}^{(i)}\right) \in A_{2} \times \ldots \times A_{d}$, $i=1, \ldots, N$, that avoid inconsistent patterns in time (such as default resurrections, etc.), i.e. such that

$$
0<\mathbb{P}\left(\mathbf{Z}\left(t+s_{\pi(1)}\right)=(1, \ldots, 1), \mathbf{Z}\left(t+s_{\pi(2)}\right)=\mathbf{x}_{2}^{(i)}, \ldots, \mathbf{Z}\left(t+s_{\pi(d)}\right)=\mathbf{x}_{d}^{(i)}\right) .
$$

This set of paths depends on $s_{1}, \ldots, s_{d}$, but it does not depend on $t$ by the timehomogeneity property of $\mathbf{Z}$. We have

$$
\begin{aligned}
& \mathbb{P}\left(\tau_{1}>t, \ldots, \tau_{d}>t\right) \mathbb{P}\left(\tau_{1}>s_{1}, \ldots, \tau_{d}>s_{d}\right) \\
& \quad=\mathbb{P}\left(\mathbf{Z}(t) \in A_{1}\right) \mathbb{P}\left(\mathbf{Z}\left(s_{\pi(1)}\right) \in A_{1}, \mathbf{Z}\left(s_{\pi(2)}\right) \in A_{2}, \ldots, \mathbf{Z}\left(s_{\pi(d)}\right) \in A_{d}\right) \\
& \quad=\mathbb{P}\left(\mathbf{Z}(t) \in A_{1}\right) \sum_{i=1}^{N} \mathbb{P}\left(\mathbf{Z}\left(s_{\pi(1)}\right)=(1, \ldots, 1), \mathbf{Z}\left(s_{\pi(2)}\right)=\mathbf{x}_{2}^{(i)}, \ldots, \mathbf{Z}\left(s_{\pi(d)}\right)=\mathbf{x}_{d}^{(i)}\right) \\
& \quad=p_{(1, \ldots, 1),(1, \ldots, 1)}(t) \sum_{i=1}^{N} p_{(1, \ldots, 1),(1, \ldots, 1)}\left(s_{\pi(1)}\right) p_{(1, \ldots, 1), \mathbf{x}_{2}^{(i)}}\left(s_{\pi(2)}-s_{\pi(1)}\right) \prod_{k=3}^{d} p_{\mathbf{x}_{k-1}^{(i)}, \mathbf{x}_{k}^{(i)}}\left(s_{\pi(k)}-s_{\pi(k-1)}\right) \\
& \quad=\sum_{i=1}^{N} p_{(1, \ldots, 1),(1, \ldots, 1)}\left(t+s_{\pi(1)}\right) p_{(1, \ldots, 1), \mathbf{x}_{2}^{(i)}}\left(t+s_{\pi(2)}-\left(t+s_{\pi(1)}\right)\right) \prod_{k=3}^{d} p_{\mathbf{x}_{k-1}^{(i)}, \mathbf{x}_{k}^{(i)}}\left(t+s_{\pi(k)}-\left(t+s_{\pi(k-1)}\right)\right) \\
& \quad=\mathbb{P}\left(\mathbf{Z}\left(t+s_{\pi(1)}\right) \in A_{1}, \mathbf{Z}\left(t+s_{\pi(2)}\right) \in A_{2}, \ldots, \mathbf{Z}\left(t+s_{\pi(d)}\right) \in A_{d}\right) \\
&= \mathbb{P}\left(\tau_{1}>t+s_{1}, \ldots, \tau_{d}>t+s_{d}\right)
\end{aligned}
$$

Repeating the above derivation for every subset $I \subset\{1, \ldots, d\}$ we obtain the equation

$$
\mathbb{P}\left(\tau_{i_{1}}>t+s_{i_{1}}, \ldots, \tau_{i_{k}}>t+s_{i_{k}}\right)=\mathbb{P}\left(\tau_{i_{1}}>t, \ldots, \tau_{i_{k}}>t\right) \mathbb{P}\left(\tau_{i_{1}}>s_{i_{1}}, \ldots, \tau_{i_{k}}>s_{i_{k}}\right)
$$

for arbitrary $1 \leq i_{1}, \ldots, i_{k} \leq d$ and $t, s_{i_{1}}, \ldots, s_{i_{k}} \geq 0$. This is precisely the functional equation describing the multi-variate lack-of-memory property, which is well-known to characterize the Marshall-Olkin exponential distribution, see [Marshall, Olkin (1967), Marshall, Olkin (1995)]. 
" $\Rightarrow$ " Assume $\left(\tau_{1}, \ldots, \tau_{d}\right)$ has a Marshall-Olkin distribution with parameters $\left\{\lambda_{I}\right\}, \emptyset \neq$ $I \subset\{1, \ldots, d\}$ satisfying $\sum_{I: k \in I} \lambda_{I}>0$ for all $k=1, \ldots, d$. We prove Markovianity of $\mathbf{Z}_{I}$ for an arbitrary non-empty subset $I$ of components. Without loss of generality, we may assume that $\left(\tau_{1}, \ldots, \tau_{d}\right)$ is defined on the following probability space, as first considered in [Arnold (1975)]: we consider an iid sequence $\left\{E_{n}\right\}_{n \in \mathbb{N}}$ of exponential random variables with rate $\lambda:=\sum_{\emptyset \neq K \subset\{1, \ldots, d\}} \lambda_{K}$ and an independent iid sequence $\left\{Y_{n}\right\}_{n \in \mathbb{N}}$ of set-valued random variables with distribution given by

$$
\mathbb{P}\left(Y_{1}=K\right)=\frac{\lambda_{K}}{\lambda}, \quad \emptyset \neq K \subset\{1, \ldots, d\} .
$$

The random vector $\left(\tau_{1}, \ldots, \tau_{d}\right)$ is then defined as $\tau_{k}:=E_{1}+\ldots+E_{\min \left\{n: k \in Y_{n}\right\}}, k=$ $1, \ldots, d$. Let us introduce the notation

$$
N_{t}:=\sum_{k=1}^{\infty} \mathbf{1}_{\left\{E_{1}+\ldots+E_{k} \leq t\right\}}, \quad t \geq 0
$$

which is a Poisson process with intensity $\lambda$. Fix a non-empty set $I \subset\{1, \ldots, d\}$, say $I=\left\{i_{1}, \ldots, i_{k}\right\}$ with $1 \leq i_{1}<\ldots<i_{k} \leq d$. Denoting the power set of $\{1, \ldots, d\}$ by $\mathcal{P}_{d}$, we define the function $f_{I}:\{0,1\}^{k} \times \mathcal{P}_{d} \rightarrow\{0,1\}^{k}$ as follows:

$$
j \text {-th component of } f_{I}(\vec{x}, J):=\mathbf{1}_{\left\{x_{j}=1 \text { and } i_{j} \notin J\right\}}, \quad j=1, \ldots, k,
$$

for $\vec{x}=\left(x_{1}, \ldots, x_{k}\right) \in\{0,1\}^{k}$ and $J \in \mathcal{P}_{d}$. It is now readily observed - in fact just a rewriting of Arnold's model - that

$$
\mathbf{Z}_{I}(t)=f_{I}\left(\mathbf{Z}_{I}(s), \bigcup_{k=N_{s}+1}^{N_{t}} Y_{k}\right), \quad t \geq s \geq 0 .
$$

This stochastic representation implies the claim, since the second argument of $f_{I}$ is independent of $\mathcal{F}_{I}(s):=\sigma\left(\mathbf{Z}_{I}(u): u \leq s\right)$ by the Poisson property of $\left\{N_{t}\right\}$. To see this, it suffices to observe that $Z_{I}(s)$ is a function of $N_{s}$ and $Y_{1}, \ldots, Y_{N_{s}}$ (which can be seen by setting $t=s$ and $s=0$ in (3)), whereas the second argument is a function of $Y_{N_{s}+1}, \ldots, Y_{N_{t}}$. Consequently, the independent random variables $N_{s}$ and $N_{t}-N_{s}$ only serve as a random pick of two independent (because disjoint) partial sequences of the iid sequence $Y_{1}, Y_{2}, \ldots$

\section{Practical restrictions for multivariate default models}

Connections between Markov chains and random vectors have already been studied in the literature. A very general framework is given by the multivariate phase-type distributions family (MPH). This has been thoroughly developed in the late seventies by M.F. Neuts and co-workers, a survey can be found in [Bladt (2005)]. MPH are introduced in the context of default risk in [Assaf et al. (1984)], see also [Cai, Li (2005)], 


\section{Practical restrictions for multivariate default models}

defining a random vector explicitly via a Markov chain. Unfortunately, multi-variate phase type distributions, due to their generality, appear to be very difficult to work with in high-dimensional applications. A more practical, proper subclass of MPH is given by the family of default times whose associated survival indicator process $\boldsymbol{Z}$ is a continuoustime Markov chain. This family, which we abbreviate by $\mathrm{MCH}$, has been studied extensively in the context of credit-risk modeling in [Herbertsson, Rootzén (2008), Bielecki et al. (2011b)], and includes looping defaults, and thus default contagion in the sense of [Jarrow, Yu (2001), $\mathrm{Yu}$ (2007)]. However, the MCH family still imposes serious challenges in a real world implementation, since it is not naturally equipped with a "nested margining property" (cf. (P2) below). The main practical implication of Theorem 2.2 is that the latter property is satisfied by $\mathrm{MO}$, and moreover, $\mathrm{MO}$ is actually characterized by this property within the MCH family. Acknowledging previous results, it is known since [Assaf et al. (1984)] that $\mathrm{MO} \subsetneq \mathrm{MPH}$, and it is also not difficult to observe that $\mathrm{MO} \subsetneq \mathrm{MCH}$. To the best of our knowledge, however, existing literature did neither realize that MO is essentially the only family of distributions satisfying the nested margining property, nor did it address the economic consequences thereof.

We describe two practical aspects that manifest severe problems when a portfolio-default model is integrated into a financial institution's existing simulation engine, which nowadays is needed for the assessment of risk measures or counterparty exposures. In particular, these issues limit the modeling cosmos and justify the use of MO as portfolio-default model.

(P1) Stepwise simulation: A global simulation of a large portfolio, possibly consisting of different asset classes and risk factors, naturally requires diverse knowledge. In particular, different specialized departments deliver their sub-portfolio models to a dedicated department which aggregates these models to a global one. One common ground for the aggregation of several different models to one global simulation engine is typically the time grid, which serves as a basis for the propagation of all risk factors in all models. Furthermore, the mesh of this simulation time grid must be variable, since regulation suggests the use of different holding periods in the assessment of risk measures, see, e.g., [BIS Consultative Document (2012)]. As a consequence for the portfolio-default model this necessitates the need to simulate paths of the process $\boldsymbol{Z}$ stepwise, along a given time grid with all other (e.g. Brownian-driven) risk factors. This is a critical issue, since many copulabased models that are currently in use lack a natural coherence with stepwise simulation. Generally speaking, we face the task of simulating a path of $\mathbf{Z}$ along an equidistant grid with length $\Delta$, the smallest common time step needed to reach all the desired grid times, i.e. the sequence $(\mathbf{Z}(0), \mathbf{Z}(\Delta), \mathbf{Z}(2 \Delta), \ldots)$. In order to carry out the simulation in a stepwise manner, in step $k$ of the simulation we have to simulate $\mathbf{Z}(k \Delta)$ from the discrete distribution

$$
\left(P_{\mathbf{Z}((k-1) \Delta), h(J)}\left[(k-1), \mathcal{F}_{(k-1) \Delta}\right]\right)_{J \subset\{1, \ldots, d\}},
$$




\section{Practical restrictions for multivariate default models}

where

$$
P_{h(I), h(J)}\left[(k-1), \mathcal{F}_{(k-1) \Delta}\right]:=\mathbb{P}\left(\mathbf{Z}(k \Delta)=h(J) \mid\left(\mathbf{Z}((k-1) \Delta)=h(I), \mathcal{F}_{(k-1)} \Delta\right),\right.
$$

with $\mathcal{F}_{t}$ being the $\sigma$-algebra of all available information at time $t$. For global simulations in practical tasks it is a convenient simplification to restrict one's attention to models in the MCH class, i.e. in which $\mathbf{Z}$ is a continuous-time Markov chain. In particular, this implies that

$$
P_{h(I), h(J)}\left[(k-1), \mathcal{F}_{(k-1) \Delta}\right]=P_{h(I), h(J)}[\Delta]
$$

depends only on $\Delta$. The ability for efficient stepwise simulation along a given time grid stems from the fact that continuous-time Markov chains can be discretized to discrete-time Markov chains via the computation of the matrix exponential of their associated generator matrix, leading to easily implementable transition matrices. Furthermore, the articles [Herbertsson, Rootzén (2008), Bielecki et al. (2011b)] demonstrate that the resulting $\mathrm{MCH}$ family of default models is flexible and tractable enough to efficiently price portfolio-credit derivatives with static composition. However, MCH models do not necessarily exhibit the following nested margining property, which has enormous practical implications.

(P2) Nested margining property: If one wishes to carry out a simulation study involving only a distinct sub-portfolio, i.e. a subgroup $I \subsetneq\{1, \ldots, d\}$ of components, in general one still has to simulate the whole portfolio $\mathbf{Z}$ and cannot simulate the subvector-indicator process $\mathbf{Z}_{I}$ directly with a more efficient simulation engine. Hence, it is a priori not guaranteed that the model is stable under taking margins, called "nested margining property". One would like to be able to design the model by means of a hierarchical factor-loading way of thinking, which is particularly crucial for large portfolios that are frequently restructured. If the application in concern requires us to add (remove) components to (from) our portfolio on a frequent basis, every such change might alter the dependence structure between the original components, and therefore requires careful and potentially very time consuming readjustments of the model. In other words, one would like to be able to increase or reduce the model dimension straightforwardly. These frequent readjustments are much easier to understand and implement in case of an available hierarchical structure consisting of groups to which new (from which old) components are added (removed). Models with the property of being consistent when varying their dimension are very manageable and popular. A typical nested-margining representative is the Gaussian copula model with hierarchical, multi-factor parameterisation of its correlation matrix, that is, however, not part of the MO class, and violating (P1) and (P2).

Within the MCH family, the subfamily of models for which every subvector indicator process $\mathbf{Z}_{I}$ is a continuous-time Markov chain satisfies the nested margining property. This is because Markovianity of $\boldsymbol{Z}_{I}$ guarantees that each process $\mathbf{Z}_{I}$ can be simulated without the need to know the full history of $\boldsymbol{Z}$. Theorem 2.2 


\section{Parameterization and efficient implementation}

shows that the subfamily $\mathrm{MO} \subsetneq \mathrm{MCH}$ is characterized precisely by that property. Furthermore, Section 4 illustrates how factor-model constructions and efficient implementation are intimately linked in case of the Marshall-Olkin distribution via the so-called "Lévy-frailty construction".

Let us close this section with two considerations. Firstly, we recall the important stylized statistical facts of the MO law. It extends the idea of the exponential law, i.e. default times with constant intensity, to higher dimensions. Another prominent feature is the singular component - allowing for joint defaults / cataclysmic events. The related MO copula belongs to the class of extreme-value copulas and, hence, is naturally capable of modeling tail dependence. For practical applications, the intuitive parameterisation is generally seen as an advantage, although the interpretation via exogeneous shocks (2) comes at the cost of numerical difficulties, since the number of shocks grows exponentially in $d$. This challenge is overcome in the following section. Lastly, we need to pay attention in distinguishing the MO multivariate distribution from its copula. A multivariate distribution given by a MO copula with re-scaled exponential margins does not necessarily satisfy Theorem 2.2. In other terms, we need the whole multivariate MO distribution with its natural consistency between margins and dependence parameters, see for example [Mai, Scherer (2013)]. The MO distribution consistency avoids problems like deterministic ordering of default times in presence of deterministic intensities and the related issues in wrong way risk modeling, see for example the vanishing wrong way risk case study for default modeling in counterparty risk valuation (CVA) in [Brigo, Chourdakis (2009)].

\section{Parameterization and efficient implementation}

The subject of this section is the efficient implementation of an unbiased simulation scheme for the Marshall-Olkin law. More details are given in [Brigo et al. (2014)]. We consider the tasks:

(a) Finding a convenient parameterization of the Marshall-Olkin law, especially in large dimensions.

(b) Constructing an efficient and unbiased simulation engine for the Marshall-Olkin law along a given time grid $0<t_{1}<t_{2}<\ldots<t_{n}=T$.

Simulation requires a (preferably simple) stochastic model. There exist two classical stochastic representations for the Marshall-Olkin law. The one of [Marshall, Olkin (1967)], see (2), requires $2^{d}-1$ exponentially distributed shocks. The second from [Arnold (1975)], see (3), is based on compound sums of exponentials. In both models, the tasks (a) and (b) are intimately linked, because the number of parameters different from zero enters the (expected) runtime of the respective simulation algorithms, see [Mai, Scherer (2012b), Chapter 3.1]. Many references tackle this issue by setting most parameters to zero, see for example [Giesecke (2003), Lindskog, McNeil (2003)]. However, this results in simplistic subfamilies. Concluding, these canonical stochastic models are not recommended 


\section{Parameterization and efficient implementation}

in dimensions greater than, say, $d=10$, although occasionally the dynamical properties of the aggregated default counting process and of the related loss distribution have been studied under pool homogeneity assumptions in dimensions as large as $d=125$, see for example [Brigo et al. (2007)] where the first calibration to CDO market data under an arbitrage free dynamic loss model inspired by a bottom-up Marshall-Olkin approach is presented. We should also mention that, in this context, [Bielecki et al. (2011a)] manage to refine such results and attain high dimensions and realistic calibration to market data while modeling defaults in a bottom-up fashion and with no need to assume pool homogeneity.

There exists, however, a third stochastic representation of the Marshall-Olkin distributions due to [Mai, Scherer (2009), Mai, Scherer (2011)], which has been generalized and applied to portfolio-credit risk by [Sun et al. (2012)]. Based on the notions of Lévy subordinators, it is called "Lévy-frailty construction", and - since it is just an alternative representation for the Marshall-Olkin law - it satisfies the practical requirements (P1) and (P2) of Section 3. With regards to the tasks (a) and (b), it has two crucial advantages:

(1) A simulation along a given time grid is natural and straightforward. The numerical effort increases only linearly in the dimension $d$ and the number of time steps of the grid.

(2) The number of parameters does not depend on the dimension, but instead can be chosen quite arbitrarily. Moreover, the stochastic model can be interpreted as a factor model.

Starting from $m$ independent Lévy subordinators $\hat{\Lambda}^{(1)}, \ldots, \hat{\Lambda}^{(m)}$ with Laplace exponents $\hat{\Psi}_{1}, \ldots, \hat{\Psi}_{m}$ and considering the weight vectors $\boldsymbol{\theta}_{i} \in \mathbb{R}_{+}^{m}, i=1, \ldots, d$, we can define the $d$-dimensional subordinator $\boldsymbol{\Lambda}=\left(\Lambda^{(1)}, \ldots, \Lambda^{(d)}\right)$, where $\Lambda^{(i)}=\boldsymbol{\theta}_{i}^{\prime} \hat{\boldsymbol{\Lambda}}$. The random vector $\left(\tau_{1}, \ldots, \tau_{d}\right)$, defined via

$$
\tau_{i}:=\inf \left\{t \geq 0: \Lambda_{t}^{(i)} \geq \epsilon_{i}\right\}, \quad i=1, \ldots, d,
$$

with independent unit-mean exponential random variables $\epsilon_{1}, \ldots, \epsilon_{d}$, independent of $\boldsymbol{\Lambda}$, has a Marshall-Olkin distribution, cf. [Sun et al. (2012), Mai (2014)]. Stepwise simulation is natural within this model, as Algorithm 1 shows.

\section{Algorithm 1 (Sampling the Lévy-frailty model on a given time grid)}

Given the time grid $0=t_{0}<t_{1}<\ldots<t_{n}=T$ and the Lévy subordinator $\boldsymbol{\Lambda}$, initialize the current time as $t^{*}:=0, \ell:=0$, and the number of components that are still alive by nalive $=d$.

(1) Repeat the following steps until ((nalive $==0)$ or $\left(t^{*}==T\right)$ ), i.e. until all components have defaulted or the final time horizon is reached, whichever takes place first:

(a) Set $t^{*}:=t_{\ell+1}$. 


\section{Conclusion}

(b) Simulate the next increment $\Delta \boldsymbol{\Lambda}_{\ell}:=\boldsymbol{\Lambda}_{t_{\ell+1}}-\boldsymbol{\Lambda}_{t_{\ell}} \sim \boldsymbol{\Lambda}_{t_{\ell+1}-t_{\ell}}$ of the multivariate Lévy subordinator on the time interval $\left[t_{\ell}, t_{\ell+1}\right]$. Note that this is independent of the past, by the Lévy properties of $\boldsymbol{\Lambda}$.

(c) Simulate a list of independent unit exponentials $\epsilon_{i_{1}}, \ldots, \epsilon_{i_{\text {nalive }}}$ for the components $\tau_{i_{1}}, \ldots, \tau_{i_{\text {nal ive }}}$ that have not yet defaulted. This is justified by the lack-of-memory property of the unit exponential law, i.e. the positive distance of a trigger variate (that has not yet defaulted) to the current state of the subordinator has a unit exponential law.

(d) For each $\epsilon_{i_{k}}, k=1, \ldots$, nalive, test if $\left(\Delta \Lambda_{\ell}^{\left(i_{k}\right)}>E_{i_{k}}\right)$. Each time this condition is met, set $\tau_{i_{k}}:=t^{*}$ and decrease nalive by one. ${ }^{1}$

(e) $\ell:=\ell+1$.

(2) Return the vector $\left(\tau_{1}, \ldots, \tau_{d}\right) \in\left\{t_{0}, \ldots, t_{n}\right\}^{d}$ or, equivalently, the path of the indicator process $\left(\mathbf{1}_{\left\{\tau_{1}>t\right\}}, \ldots, \mathbf{1}_{\left\{\tau_{d}>t\right\}}\right)$ sampled on the given time grid.

It is also clear that the parameters of the resulting Marshall-Olkin distribution are functions of the parameters of the involved Lévy subordinators $\hat{\Lambda}^{(1)}, \ldots, \hat{\Lambda}^{(m)}$, which are themselves parameterized in terms of their respective Laplace exponents. This allows to control the involved number of model parameters irrespectively of the dimension $d$. Furthermore, hierarchical factor-loading constructions, e.g. according to regional or industry-specific categories, are possible by interpreting the independent stochastic drivers $\hat{\Lambda}^{(1)}, \ldots, \hat{\Lambda}^{(m)}$, and by choosing the weights accordingly. See, e.g., [Mai, Scherer (2012a)] for a recipe on how to construct hierarchical factor structures in a Marshall-Olkin distribution.

\section{Conclusion}

The postulate that all sub-vectors of some survival indicator process are continuoustime Markov chains constitutes a new characterization of the Marshall-Olkin law. It is demonstrated that this result has important economic implications, as it renders the Marshall-Olkin law the only class of portfolio-default models satisfying a list of important practical demands. An efficient and unbiased simulation scheme, based on a multi-factor Lévy-frailty construction, provides methodological advice for the actual implementation of an economic scenario generator.

\footnotetext{
${ }^{1}$ Instead of drawing exponential random variables along the lines of the Lévy-frailty model, one might instead use Bernoulli $\left(1-\exp \left(-\Delta \Lambda_{\ell}^{\left(i_{k}\right)}\right)\right)$ distributed ones in Step (1) (c,d) of Algorithm 1. This is justified by the observation that the conditional default probability of $\tau_{k}$ within $\left[t_{\ell}, t_{\ell+1}\right]$ given $\Delta \Lambda_{\ell}^{\left(i_{k}\right)}$ is precisely $1-\exp \left(-\Delta \Lambda_{\ell}^{\left(i_{k}\right)}\right)$. If the respective Bernoulli experiment was successful, component $k$ defaults and its default time is set to $\tau_{k}:=t^{*}$.
} 


\section{References}

\section{References}

[Arnold (1975)] B.C. Arnold, A characterization of the exponential distribution by multivariate geometric compounding, Sankhyā: The Indian Journal of Statistics 37(1) (1975) pp. 164-173.

[Assaf et al. (1984)] D. Assaf, N. Langberg, T. Savits, M. Shaked, Multivariate phasetype distributions, Operations Research 32 (1984) pp. 688-702.

[BIS Consultative Document (2012)] BIS Consultative Document, Fundamental review of the trading book, Annex 4, available at BIS.org (2012).

[Bielecki et al. (2011a)] T.R. Bielecki, A. Cousin, S. Crépey, A. Herbertsson, Dynamic modeling of portfolio credit risk with common shocks. Available at ssrn.com (2011).

[Bielecki et al. (2011b)] T.R. Bielecki, S. Crépey, A. Herbertsson, Markov chain models of portfolio credit risk, in The Oxford Handbook of credit derivatives, edited by $A$. Lipton, A. Rennie, Oxford University Press (2011) pp. 327-382.

[Bladt (2005)] M. Bladt, A review on phase-type distributions and their use in risk theory, ASTIN Bulletin 35 (2005) pp. 145-161.

[Brigo, Chourdakis (2009)] D. Brigo, K. Chourdakis, Counterparty Risk for Credit Default Swaps: Impact of spread volatility and default correlation. International Journal of Theoretical and Applied Finance 12 (7) (2009), pp. 1007-1026.

[Brigo, Chourdakis (2012)] D. Brigo, K. Chourdakis, Consistent single- and multi-step sampling of multivariate arrival times: A characterization of self-chaining copulas. Available at arXiv.org, ssrn.com, defaultrisk.com (2012).

[Brigo et al. (2014)] D. Brigo, J.F. Mai, and M. Scherer (2014). Consistent iterated simulation of multi-variate default times: a Markovian indicators characterization. Preprint, available at SSRN.com and arXiv.org

[Brigo et al. (2013)] D. Brigo, M. Morini, A. Pallavicini, Counterparty Credit Risk, Collateral and Funding, with Pricing Cases for all Asset Classes, Wiley (2013).

[Brigo et al. (2007)] D. Brigo, A. Pallavicini, R. Torresetti, Cluster-based extension of the generalized Poisson loss dynamics and consistency with single names, International Journal of Theoretical and Applied Finance, 10(4) (2007), also in A. Lipton and Rennie (Editors), Credit Correlation - Life After Copulas, World Scientific, 2007.

[Cai, Li (2005)] J. Cai, H. Li, Conditional tail expectations for multivariate phase type distributions, working paper (2005).

[Giesecke (2003)] K. Giesecke, A simple exponential model for dependent defaults, Journal of Fixed Income 13(3) (2003) pp. 74-83. 


\section{References}

[Herbertsson, Rootzén (2008)] A. Herbertsson, H. Rootzén, Pricing $k$-th-to-default Swaps under Default Contagion: The Matrix-Analytic Approach, The Journal of computational Finance 12 (2008) pp. 49-72.

[Jarrow, Yu (2001)] R. Jarrow, F. Yu, Counterparty risk and the pricing of defaultable securities, Journal of Finance 56 (2001) pp. 1765-1800.

[Lindskog, McNeil (2003)] F. Lindskog, A.J. McNeil, Common Poisson shock models: Applications to insurance and credit risk modelling, Astin Bulletin 33(2) (2003) pp. 209-238.

[Mai (2014)] J.-F. Mai, Multivariate exponential distributions with latent factor structure and related topics, Habilitation thesis, Technische Universität München, https://mediatum.ub.tum.de/node?id=1236170 (2014).

[Mai, Scherer (2009)] J.-F. Mai, M. Scherer, Lévy-frailty copulas, Journal of Multivariate Analysis 100(7) (2009) pp. 1567-1585.

[Mai, Scherer (2011)] J.-F. Mai, M. Scherer, Reparameterizing Marshall-Olkin copulas with applications to sampling, Journal of Statistical Computation and Simulation 81(1) (2011) pp. 59-78.

[Mai, Scherer (2012a)] J.-F. Mai, M. Scherer, H-extendible copulas, Journal of Multivariate Analysis 110 (2012) pp. 151-160.

[Mai, Scherer (2012b)] J.-F. Mai, M. Scherer, Simulating Copulas: Stochastic Models, Sampling Algorithms, and Applications, Imperial College Press (2012).

[Mai, Scherer (2013)] J.-F. Mai, M. Scherer, What makes dependence modeling challenging? Pitfalls and ways to circumvent them, Statistics \& R Risk Modeling 30 (2013) pp. 287-306.

[Marshall, Olkin (1967)] A.W. Marshall, I. Olkin, A multivariate exponential distribution, Journal of the American Statistical Association 62 (1967) pp. 30-44.

[Marshall, Olkin (1995)] A.W. Marshall, I. Olkin, Multivariate exponential and geometric distributions with limited memory, Journal of Multivariate Analysis 53 (1995) pp. $110-125$.

[McNeil et al. (2005)] A.J. McNeil, R. Frey, P. Embrechts, Quantitative risk management, Princeton university press, Princeton, New Jersey (2005).

[Sun et al. (2012)] Y. Sun, R. Mendoza-Arriaga, V. Linetsky, Marshall-Olkin multivariate exponential distributions, multidimensional Lévy subordinators, efficient simulation, and applications to credit risk, working paper (2012).

[Yu (2007)] F. Yu, Correlated defaults in intensity-based models, Mathematical Finance 17(2) (2007) pp. 155-173. 